\title{
MENINGOCOCCAL MENINGITIS WITH PARTICULAR REFERENCE TO EPIDEMIOLOGY AND PATHOGENESIS
}

\author{
BY \\ R. W. FAIRBROTHER \\ Department of Clinical Pathology, Manchester Royal Infirmary
}

(RECEIVED FOR PUBLICATION, JULY 8, 1947)

In this country cerebrospinal meningitis (fever) is an endemic disease found mainly in children and adolescents; local outbreaks occur, but they are rare and usually involve few individuals. The disease has, however, a marked predilection for war-time conditions; relatively extensive outbreaks occurred during the 1914-18 war, and again to an even greater extent during the 1939-45 war. These outbreaks involved individuals of all ages, including Service personnel, but it is interesting to note that, even though there was a great increase in the total number of cases notified, these tended to occur singly and an epidemic, in the generally accepted sense, did not develop.

Although such general factors as the enhanced virulence of the meningococcus and the resistance of the individual are well recognized as playing an important role in the epidemiology of the disease, a precise explanation of the peculiar nature of the outbreaks is still lacking. A popular theory is that outbreaks of cerebrospinal meningitis result from a widespread meningococcal infection of the nasopharyhx in the general population (Ministry of Health Memorandum, 1940). Many workers accept the hypothesis developed by Dopter (1921) that meningococcal infection occurs in three progressive stages: (1) catarrhal, (2) septicaemic, and (3) meningitic; in the majority of cases the infection is arrested at the catarrhal stage and the occurrence of meningitis is a rare phenomenon (Lundie and others, 1915; Herrick 1918; Murray, 1929; Brinton, 1941). The evidence offered to support this hypothesis is, however, inconclusive and unconvincing. Furthermore this theory does not provide a solution of many problems presented in the various outbreaks, and it seemed desirable that the question should be re-examined in the light of information gained during the 1940-42 outbreak.
During the past seven years an opportunity has been available of studying the disease under Service and civilian conditions during both so-called epidemic and interepidemic periods and it was possible to direct attention particularly to the epidemiology and pathogenesis of the disease. The results are discussed below; they do not support the above hypothesis and an alternative is offered.

\section{Technique}

Cerebrospinal fluid.-Samples of cerebrospinal fluid were centrifuged as soon after collection as possible for 5 or 10 minutes; the supernatant fluid was decanted and the deposit seeded heavily on to Loeffler serum slopes and blood-agar plates, films were then made and the remainder of the deposit added to $10 \mathrm{ml}$. of glucosebroth, usually containing a small amount of paraaminobenzoic acid. Incubation was carried out at $37^{\circ} \mathrm{C}$.; the blood-agar plates were usually placed in an atmosphere containing approximately 10 per cent carbon dioxide.

Nasopharyngeal swabs.-Material was collected from the nasopharynx by means of the West swab, which was pressed and then stroked firmly against the mucous membrane. Cultures were prepared on blood-agar plates; after overnight incubation under ordinary atmospheric conditions, suspicious colonies were subcultured on to Loeffler slopes for further investigation; the plates were invariably incubated a further twenty-four hours and re-examined.

Blood cultures.-Ten $\mathrm{ml}$. of blood were collected by venepuncture; $2 \mathrm{ml}$. were added to a small amount of trypsin broth before mixing with $10 \mathrm{ml}$. agar, $2 \mathrm{ml}$. to Robertson's meat medium, and the remainder was placed in 25 or $50 \mathrm{ml}$. of glucose broth. Incubation at $37^{\circ} \mathrm{C}$. was continued for seven to fourteen days before a negative report was given, subcultures on Loeffler serum slopes being made after forty-eight hours and later as indicated.

The identification of the meningococcus was carried out by fermentation tests with sucrose, maltose, and 
glucose in Hiss's serum water and finally by agglutination tests using Standard Group sera. The suspensions for the agglutination tests were made by emulsifying the growth from a Loeffler slope in normal saline and adjusting to a light opacity; the tube method with incubation at $55^{\circ}$ C. was used. Standard Group sera, from Army and M.R.C. sources, were used throughout.

Preliminary tests were frequently carried out by the slide agglutination technique; useful information was obtained when the strains readily agglutinated, but this method proved unsatisfactory with relatively inagglutinable organisms.

\section{Scope of the Investigation}

Material was collected from various sources during this investigation, particularly from cases, close contacts of cases, and members of the general community (Table I).

TABLE I

SOURCE OF MATERIAL

\begin{tabular}{cccc|c}
\hline \multicolumn{3}{c|}{ Type of case } & Number \\
\hline Meningitis $\begin{array}{llll}\text { service } \\
\text { civilian }\end{array}$ & $\ldots$ & $\ldots$ & $\ldots$ & 105 \\
\hline Purulent conjunctivitis & $\ldots$ & $\ldots$ & $\ldots$ & 2 \\
\hline Chronic septicaemia & $\ldots$ & $\ldots$ & $\ldots$ & 3 \\
\hline ? Carrier & & & & \\
$\quad \begin{array}{l}\text { close contacts } \\
\text { general community }\end{array}$ & $\ldots$ & $\ldots$ & $\ldots$ & 144 \\
\end{tabular}

Cerebrospinal fluid only was examined from the civilian cases of meningitis, but the Service cases were investigated in greater detail. Cerebrospinal fluid was examined from all, while blood cultures and nasopharyngeal swabs were collected from a limited number. The search for carriers was carried out by the collection of nasopharyngeal swabs. Close contacts (Service personnel) were considered to be individuals occupying beds adjoining the case or all occupants of small rooms or tents in which the case was sleeping (War Office Memorandum, 1940). While such a definition did not embrace all close contacts, it did ensure that all members of this group had been exposed to the nasopharyngeal discharge of the patient a short time before the collection of the swab.

\section{Clinical Course and Therapy}

The various clinical manifestations of meningococcal infections have been described in detail by Rolleston (1919), Priest (1941), and Brinton (1941). The cases seen during this investigation followed the general pattern. The cases of meningitis during the 1940-42 outbreak were mainly of a severe type, the initial symptoms being headache, neck rigidity, vomiting, and malaise. Some examples of the fulminating meningeal type were seen; these within a few hours of the onset of the disease exhibited mental confusion and delirium. During the later stages of the outbreak the mild abortive form of the disease was occasionally encountered. No examples of adrenal involvement (WaterhouseFriderichsen syndrome) or of encephalitis (Banks and McCartney, 1942) were seen.

Intensive chemotherapy with a sulphonamide, usually sulphapyridine, was begun immediately the diagnosis was established on the lines recommended by Banks (1939). Serum was not used. The usual practice was to examine the cerebrospinal fluid before chemotherapy was instituted; the fluid was invariably turbid and meningococci generally demonstrable.

In the fulminating types of disease the soluble form of the sulphonamide was administered by the intravenous route.

The results were excellent; in the group of 105 Service cases there was only one death. This is very much lower than the general mortality rate for the disease, but it must be appreciated that the cases were adults of military age and that therapy was usually instituted early in the disease; it is well known that the mortality rate is highest at the extremes of life (Jubb, 1943; Beeson and Westerman, 1943).

Several cases of chronic meningococcal septicaemia were suspected on clinical grounds, but in only three instances was it possible to confirm the diagnosis by blood culture. When this was accomplished sulphonamide therapy was instituted with the anticipated dramatic effect.

\section{Bacteriology}

Meningococci isolated during this investigation generally behaved in the characteristic manner which was described in detail by Murray (1929). A few interesting features were, however, observed, and these merit special consideration.

The standard solid media used throughout the invéstigation were 6 per cent blood-agar plates and Loeffler serum slopes. Both media gave a satisfactory growth but it was observed that, under ordinary atmospheric conditions, strains isolated in pure culture directly from the cerebrospinal fluid grew readily on the Loeffler serum slopes in screw-cap bottles, while growth on blood-agar plates was not infrequently poor; incubation of the blood agar plate in 10 per cent carbon dioxide, however, gave a luxurious growth. It therefore follows 
that, if blood-agar is used for the isolation of the meningococcus from cerebrospinal fluid, incubation should be carried out in an atmosphere containing carbon dioxide.

Biochemical tests were limited to the fermentation of glucose, maltose, and sucrose in serum-water. The great majority of strains gave consistent results, glucose and maltose being fermented but not sucrose; irregularities were occasionally found.

The agglutination tests followed the generally accepted rule that strains isolated during an "epidemic" period agglutinate readily with standard sera, but during "non-epidemic" periods inagglutinable strains tend to appear. During the main 1940-42 outbreak, cerebrospinal fluid strains agglutinated readily with the Standard Group sera; many gave clear-cut agglutination only with Group I serum, but some did not exhibit any marked antigenic specificity and agglutinated also to varying degrees with Group II serum. Many indications of the complex antigenic structure of the meningococcus were obtained during this investigation, but it was not possible to examine this problem. A simple classification by means of Standard Group sera only was attempted.

When the large outbreak had subsided and infrequent sporadic cases only were reported, a different serological picture was seen. Thus during the 1946-7 winter several severe cases, mainly in infants, were investigated; meningococci were isolated from the cerebrospinal fluid but these tended to agglutinate poorly, usually giving a slight reaction only with the Group II serum; one strain proved to be inagglutinable with the sera available (Table II).

Nasopharyngeal swabs gave similar results; strains isolated during the main outbreak were more easily grouped than those obtained during the non-epidemic period. Throughout the investigation Group II strains were predominant.

\section{Epidemiology}

The epidemiology of cerebrospinal meningitis presents several unusual features for which no adequate explanation has been offered. The marked predilection of the disease for war-time conditions has long been recognized, and therefore the sudden widespread outbreak of the disease at the beginning of 1940 was not unexpected. It is interesting to note that the incidence of the disease in this country during 1940 reached the unprecedented total of 12,771 cases; this figure is actually greater than the total figures for the 1914-18 period. There was not, however, an epidemic in the generally accepted sense of the term; cases were widely distributed, occurred sporadically, and involved individuals of all ages. Fortunately the sulphonamides proved excellent therapeutic agents and some of the serious problems of 1914-18, such as the high mortality rate and long periods of hospitalization, did not arise.

During the 1914-18 outbreak special research teams carried out extensive investigations of the disease and the following important fundamental points were established: many normal individuals harbour the meningococcus in their nasopharynx; cases of cerebrospinal meningitis develop almost entirely from contact with a carrier (instances of infection from other cases of the disease occur but they are relatively rare); the disease is transmitted by droplet infection, and consequently overcrowding and poor ventilation play prominent roles in the spread of infection.

The precise significance of the carrier is uncertain. Glover (1920) claimed that a carrier rate of over 20 per cent in a community was not only an index of overcrowding but also a warning of an impending outbreak of meningitis, and he stressed the importance of the search for carriers as a prophylactic measure. Subsequent observations have, however, failed to confirm this rather extreme view. Dudley and Brennan (1934) in a naval garrison found a carrier rate of 13 per cent associated with 11 cases of meningitis, while at a later period the carrier rate was 55 per cent but no case of meningitis developed. Other workers have also found carrier rates over 20 per cent without any increased incidence of meningitis (Straker et al., 1939). It is, therefore, obvious that the development of meningitis in an individual is dependent on factors other than the presence of carriers in the community, and there is little doubt that these are closely concerned with (1) the virulence of the meningococcus, and (2) the resistance of the host.

Virulence of meningococcus.-The absence of suitable animals has prevented a detailed study of this problem and it has been necessary to depend mainly on indirect serological tests for information. During the 1914-18 outbreak four serological types were identified (Gordon and Murray, 1915); these types were not sharply demarcated and considerable antigenic overlapping occurred. In view of this other workers (Griffith, 1918; Scott, 1918) considered that the most practical serological classification was into two main groups-Group I embracing types I and I.II, and Group II comprising types II and IV. It was generally recognized that inagglutinable strains were particularly prominent in the nasopharynx during interepidemic periods.

The use of Group sera is now the accepted method of subdividing meningococci, but knowledge of the antigenic structure of the meningococcus is still incomplete and irregularities are not uncommon. 
In the 1914-18 outbreak, the majority of cases were caused by types I and II, but since that time there has been a close relationship between Group I strains and major outbreaks. It is therefore not surprising to find that, while Group II strains have predominated in carriers, some 90 per cent of strains isolated in this country from cerebrospinal fluid during the 1940-42 outbreak belonged to Group I (Harries, 1942; Ministry of Health Report, 1946).

During this investigation there was striking serological difference in the strains isolated during the 1940-42 outbreak and those isolated from the infrequent cases seen during the 1946-7 winter (Table II).

TABLE II

SEROLOGY OF MENINGOCOCCI ISOLATED FROM CEREBROSPINAL FLUID

\begin{tabular}{ccccc|c|c}
\hline & & & & & $1940-2$ & $1946-7$ \\
\hline Group I &.. &. &.. &.. & 86 & 1 \\
\hline Group II &. &.. &.. &. & 3 & 9 \\
\hline Inagglutinable &.. &. &. & 1 & 1 \\
\hline Total &.. &.. &. &.. & 90 & 11 \\
\hline
\end{tabular}

The close association of Group I strains with major outbreaks or "epidemics" suggests strongly that such strains are more virulent than those of Group II. It is, however, the general experience that there is little or no difference in the severity of the disease produced by these strains. It must be appreciated that normal cerebrospinal fluid is an excellent culture medium for most bacteria and, provided a sufficient number is introduced, multiplication will occur irrespective of the intrinsic pathogenicity of the organism.

An important difference in the two serological groups of meningococci is therefore the greater facility with which Group I strains are able to pass from the nasopharynx to the cerebrospinal fluid. The factors concerned with the virulence of the meningococcus are obscure, but they appear to be associated with the surface, probably the capsule, of the organism. The production of toxic substances by the meningococcus has been demonstrated by various workers (Branham, 1940); knowledge of these is, however, vague, and it has not been proved that they play any part in the passage of the organism to the subarachnoid space.

The low incidence of meningitis in spite of the wide distribution of the meningococcus indicates clearly that the passage from the nasopharynx to the meninges is not readily accomplished. An important reason for this is the barrier to invasion presented by the nasopharynx; the responsible factors consequently merit close consideration.

Resistance of the host.-During the 1939-45 war conditions favoured the wide dissemination of the meningococcus, particularly in the Services, as overcrowding was common and ventilation of rooms often inadequate as a result of stringent black-out precautions. Although there was a greatly increased incidence of meningitis during the main outbreak of $1940-42$, the majority of cases were single and widely distributed, having no direct contact with each other. Most individuals would, therefore, appear to possess a high degree of resistance against the meningococcus, and the factors concerned in this have been the subject of much speculation.

Many advocate the theory that infection with the meningococcus occurs in three progressive stages: (1) catarrhal, (2) septicaemic, and (3) meningitic; the common form of the infection is a nasopharyngitis, and only in relatively few cases does the organism penetrate the pharyngeal barrier to reach the blood stream, from which the meninges are attacked. The nature of this pharyngeal barrier has, however, never been satisfactorily explained.

The so-called catarrhal stage is obviously the one of greatest epidemiological significance in view of the potential danger to the community. It is consequently important that the criteria on which this theory is postulated should be critically examined.

The main arguments put forward to support this theory are: (1) the isolation of the homologous meningococcus from both the nasopharynx and cerebrospinal fluid of early cases (Flack, 1917), (2) the history of premeningitic sore-throat or catarrh given by some cases, and (3) the widespread distribution of carriers.

These views have not passed unchallenged, and the following points have been raised in opposition: (1) the failure of many workers to isolate regularly the meningococcus from the nasopharynx of early cases of meningitis (Andrewes and others, 1916); it is also interesting to note in this connexion that Fildes and Baker (1918) reported the occurrence of meningitis in twenty-six naval ratings although their nasopharyngeal swabs had been negative at varying periods from two to seventy-five days before the onset of symptoms, and concluded that cases can seldom be carriers before the onset of the disease ; (2) a history of sore-throat or catarrh is frequently unobtainable from cases or carriers (Foster and 
Gaskell, 1916; Worster-Drought and Kennedy, 1919); (3) cerebrospinal meningitis occurs mainly during the early months of the year when upper respiratory infections are common, and their association with meningitis is likely to be coincidental (it has even' been suggested that this nonspecific catarrh might constitute a predisposing factor to the development of meningitis); (4) caseto-case infection is extremely rare; the source of infection is almost invariably an undetected carrier; (5) in the majority of instances the carrier state is temporary.

As a reply to these arguments protagonists of the catarrhal theory claim that the local meningococcal infection is generally mild and transient, giving rise to little or no personal discomfort, but clinical and bacteriological confirmation is seldom produced.

There is general agreement that in every case of meningitis the portal of entry of the meningococcus is the nasopharynx. There is, however, a lack of convincing evidence to indicate that the production of a nasopharyngitis, even in a mild form, is an essential stage in the development of meningitis. It must be pointed out that the examination of nasopharyngeal swabs from early cases is rarely carried out, and knowledge of this important aspect of the subject is consequently limited.

During this investigation the validity of the "catarrhal" theory was examined by the collection of swabs from the following groups of the community:

1. Close contacts. - These were soldiers sleeping in beds adjoining the case, or all occupants in cases arising in tents or billets. All had had ample opportunity of receiving the upper respiratory flora from the cases as overcrowding was often present and ventilation, particularly at night, usually poor; the swabs were collected within a short time, often a few hours, of the removal of the case.

2. General community.-Swabs were collected from persons having no direct contact with cases in order to obtain some indication of the general carrier rate. These have been sub-divided into two groups-one containing swabs collected at the time of the main outbreak (1940-42) and the other comprising those taken during an "interepidemic" period (1946-47).

3. Cases of meningitis.-Swabs were collected from 22 cases, in 16 of these within 48 hours of onset. Group I strains were isolated from the cerebrospinal fluid of 13 cases but no growth was obtained from the other 9,3 of which were of the mild, abortive type. The nasopharyngeal swab from one of these mild cases gave a heavy growth of a Group II strain.

4. Cases of chronic meningococcal septicaemia.-Two only were investigated.
The results obtained from these swabs are presented collectively in Table III

TABLE III

THE ISOLATION OF THE MENINGOCOCCUS FROM NASOPHARYNGEAL SWABS

\begin{tabular}{|c|c|c|c|c|}
\hline Group & $\begin{array}{c}\text { Num- } \\
\text { ber } \\
\text { exami- } \\
\text { ined }\end{array}$ & $\begin{array}{l}\text { Number } \\
\text { positive }\end{array}$ & Group I & Group II \\
\hline $\begin{array}{l}\text { Close contacts } \\
(1940-41)\end{array}$ & 145 & $21(14 \%)$ & $6(4 \%)$ & $15(10 \%)$ \\
\hline $\begin{array}{l}\text { General com- } \\
\text { munity } \\
\text { (epidemic period } \\
1940-42)\end{array}$ & 410 & $75(18 \%)$ & $11(3 \%)$ & $64(15 \%)$ \\
\hline $\begin{array}{l}\text { General com - } \\
\text { munity } \\
\text { (non-epidemic } \\
\text { period 1946-47) }\end{array}$ & 120 & $\begin{array}{c}8(7 \%) \\
.\end{array}$ & $1(1 \%)$ & $7(6 \%)$ \\
\hline $\begin{array}{l}\text { Cases of menin- } \\
\text { gitis } . .\end{array}$ & 22 & $6(27 \%)$ & $4(18 \%)$ & $2(9 \%)$ \\
\hline $\begin{array}{l}\text { Cases of chronic } \\
\text { septicaemia ... }\end{array}$ & 2 & 1 & 0 & $1^{*}$ \\
\hline
\end{tabular}

*A few colonies of a Group II meningococcus were isolated from the nasopharyngeal swab some 21 days after the onset of symptoms ; a Group I strain was isolated by blood-culture.

These results provide little bacteriological evidence to support the "catarrhal" theory. Meningococci were isolated from the nasopharynx of only 6 of 22 cases and in the majority of these the organisms were not numerous; the 1940-42 outbreak was caused almost entirely by Group I strains, but during this period the majority of carriers harboured Group II strains; there was no significant difference in the carrier rates of Group I strains given by the close contacts ( 4 per cent) and the general community ( 3 per cent), in both the rates were low; few of the cases or carriers gave a definite history of sore throat or catarrh.

The meningococcus is transmitted by droplet infection, and therefore in all persons developing meningitis these organisms must have been present in the nasopharynx during the initial stages of the infection. Their subsequent isolation from this site is dependent not only on the interval between implantation of the organisms and the collection of the swab but also on the nature of the local reaction. If a local infection develops it is reasonable to expect (1) the isolation of meningococci from the nasopharyngeal swabs in many early cases-not infrequently in practically pure culture, and (2) a relatively high carrier rate of the infecting type of 
organism among close contacts, as an interchange of the upper respiratory flora takes place readily among occupants of overcrowded and badly ventilated rooms. Such results have rarely been obtained.

It is recognized that a small number of carriers are of the "persistent" type and that the persistence of the carrier state is associated with some pharyngeal abnormality (Cleminson, 1918; Embleton and Steven, 1919). During this investigation persistent carriers were found to have some abnormality, such as enlarged adenoids, and the majority harboured Group II strains often in relatively pure culture. These organisms were usually associated with a chronic form of local infection, but they were of little epidemiological significance as the main outbreak was caused by Group I strains; they were readily cleared by a course of sulphonamides (Fairbrother, 1940).

The failure to detect the homologous meningococcus in swabs from the two chronic septicaemia cases is not surprising. Logan (1946), in an excellent review of meningococcal septicaemia, points out that the pathogenesis of the disease is uncertain; there is no bacteriological proof that the portal of entry of the organisms is the same in meningococcaemia as in meningitis, although this is probable, while a persistent focus of infection has seldom been found. In the two cases under review, the swabs were collected when the disease was well established and the negative results indicate only that the focus of the existing infection was not the nasopharynx.

\section{Pathogenesis}

The path of the meningococcus from the nasopharynx to the cerebrospinal fluid has not been conclusively determined, and in consequence it has been the subject of much speculation. Two main views have been formulated; the first accepts the direct extension of the meningococcus from the pharynx to the meninges, the second postulates that the organisms are transmitted by way of the blood stream.

The theory of direct extension was advocated during the 1914-18 outbreak but convincing proof was not produced. There has consequently been increasing support for the "haematogenous" theory, which is now generally accepted (Brinton, 1941; War Office Memorandum, 1942; Strong, 1943; Banks, 1947). It must, however, be appreciated that the available evidence to support this view is inconclusive and merits examination.

The main arguments advanced in favour of the "haematogenous" route are: (1) the development of a so-called premeningitic stage with involvement of the blood stream; (2) the rare isolation of the organism by blood-culture during the premeningitic phase while the cerebrospinal fluid was normal; (3) the isolation of the meningococcus by blood culture from early cases of meningitis; and (4) the occurrence of a septicaemic form of meningococcal infection in which there is no involvement of the meninges-these infections may occasionally be of a fulminating character.

It has already been pointed out that a satisfactory explanation has not been produced to indicate how the meningococcus enters the blood stream from the nasopharynx. Considerable doubt has, moreover, been cast on the "catarrhal" theory and there is little evidence to indicate that there is a persistent focus of infection in the nasopharynx from which the organisms pass into the blood. Also a satisfactory explanation of the method or route by which the meningococcus passes from the blood to the meninges has not been forthcoming. In this respect, it is interesting to note that there is a definite blood-central nervous system barrier; the passage of substances through the choroid plexus or other vessels into the cerebrospinal fluid is not automatic but is controlled by some form of selective mechanism. It is well known that penicillin injected into the blood does not readily reach the theca; while experimental infection with the poliomyelitis virus is extremely difficult to produce by the intravenous route, although it occurs readily by the intrathecal or intraneural route.

There is no evidence to suggest that the meningococcus has any special affinity for the choroid plexus or other part of the theca. It is indeed accepted that in most cases of meningococcal septicaemia the development of meningitis does not occur even though, in pre-sulphonamide days, the blood infection often persisted for months.

Moreover, a positive blood culture in the early and acute stages of a severe infection cannot be accepted as evidence of primary blood invasion. In such infections as pneumonia and enteric fever a positive blood culture is frequently present in the early stages as a secondary phenomenon, being an overflow of the organisms from the primary focus of the infection. It is, therefore, reasonable to claim that the positive blood culture obtained in many cases of meningococcal meningitis has been a secondary development.

A more serious argument is the rare occurrence of a positive blood culture before the development of meningitis; it must, however, be noted that in several of these cases the primary focus of infection was not established, the presence of a rhinopharyn- 
gitis being assumed, and/or the presence of meningitis was not excluded by lumbar puncture.

The occurrence of a definite premeningitic clinical stage can also be challenged. Cases of moderate severity tend to give a twenty-four-hour history of general malaise, weakness, headache of varying but usually progressive intensity, and slight neck rigidity. On lumbar puncture a turbid fluid is almost invariably obtained from these cases; this is proof that the invasion of the meninges occurred many hours previously. In fulminating meningitic cases, a turbid fluid is obtained by lumbar puncture even though the history of the infection is short, usually a matter of hours; the possibility of such infections developing in progressive stages (viz., catarrhal and septicaemic) is remote.

There is no specific sign or symptom to indicate involvement of the meninges; the initial symptoms of moderate meningeal infections are often those of an indefinite character, and such cases are not infrequently diagnosed tentatively as influenza. The presence of meningitis can be established only by an examination of the cerebrospinal fluid. The development of a premeningitic phase of the infection cannot therefore be accepted on clinical grounds alone.

In order to test the "haematogenous" theory, blood cultures were prepared as early as possible in the infection from a number of cases, usually at the time of collection of the cerebrospinal fluid and nasopharyngeal swabs. The results are given in Table IV.

TABLE IV

ISOLATION OF THE MENINGOCOCCUS FROM THE BLOOD AND CEREBROSPINAL FLUID IN CASES OF MENINGITIS

\begin{tabular}{|c|c|c|c|}
\hline $\begin{array}{l}\text { Time of } \\
\text { collection }\end{array}$ & $\begin{array}{l}\text { No. } \\
\text { examined }\end{array}$ & $\begin{array}{l}\text { No. } \\
\text { positive }\end{array}$ & $\begin{array}{l}\text { C.S.F. } \\
\text { positive in }\end{array}$ \\
\hline $\begin{array}{l}\text { Within } 24 \text { hours of } \\
\text { onset } \quad \ldots \quad \ldots\end{array}$ & 12 & 1 & 12 \\
\hline $\begin{array}{ccc}24-48 & \text { hours of on- } \\
\text { set } & \ldots & .\end{array}$ & 8 & 1 & 6 \\
\hline $\begin{array}{c}\text { Later than } \mathbf{4 8} \text { hours } \\
\text { of onset .. }\end{array}$ & 5 & 1 & 3 \\
\hline Total & 25 & 3 & 21 \\
\hline
\end{tabular}

The meningococcus was isolated from the blood stream in only 3 out of 25 cases (12 per cent). In 20 of the 25 cases the blood was collected within forty-eight hours of the onset of the disease; in all cases a. well-established meningitis was present and meningococci were isolated from the cerebrospinal fluid of 21 of the 25 .
These results are not consistent with the development of a premeningeal septicaemic stage, but indicate merely the secondary invasion of the blood stream from the primary infection of the meninges. A positive blood culture, obtained when meningitis is present and the cerebrospinal fluid contains large numbers of meningococci, cannot be accepted as evidence of either the passive transfer by the blood stream of the organisms to the subarachnoid space from an indefinite focus of infection in the nasopharynx or the presence of a distinct premeningeal phase.

It is interesting to note that the majority of positive blood-cultures from cases of cerebrospinal meningitis, as distinct from chronic septicaemia, reported in the literature have been obtained when the meningitis was well established.

\section{Discussion}

The results of this investigation do not provide evidence, clinical or bacteriological, to support the widely accepted theory that the meningococcus produces a primary pharyngeal catarrh, from which, in relatively few cases, it passes to the meninges by way of the blood stream.

This is not surprising, as evidence advanced to support this theory is far from conclusive. To account for the failure of many cases to provide a history of sore-throat or catarrh, it was suggested that the local pharyngeal infection was often transient and trivial, but bacteriological or clinical proof of this infection was seldom obtained. Moreover there has been no satisfactory explanation of the method by which the organisms enter the blood stream from such a trivial primary lesion or by which they subsequently pass from the blood stream to the meninges.

In view of the fundamental weaknesses of the haematogenous theory it is essential that an alternate method of spread, namely by direct extension. should be carefully examined. This theory received much support during the 1914-18 outbreak, when the following routes were suggested: (1) by the perineural lymphatics of the olfactory nerves through the cribriform plate of the ethmoid; (2) through the sphenoidal sinuses; (3) via the middle ear. Convincing evidence to support any of these routes was not produced, and this theory consequently became neglected.

In 1929, however, Clark produced conclusive proof that material could pass directly to the brain from the nasal passages. Investigating the development of nervous complications following vaccination, Clark showed that a solution of potassium 
ferrocyanide and iron ammonium citrate reached the subarachnoid spaces of the brain of rabbits within one hour of being dropped into their nasal cavities. The route of spread was not by the lymphatics but by the perineural sheaths of the olfactory nerves, which provide continuity between the subarachnoid space and the olfactory sensory epithelium; in these spaces a centripetal flow was postulated. It was also found that some of the solution entered the blood capillaries. On treatment of the tissues, the deposit of Prussian blue in the nasal mucosa was found to be considerably more abundant in the olfactory area, which is innervated by the olfactory nerves, than in the "respiratory" portion which is not reached by the olfactory nerves. Clark considered that a similar sequence of events, apart from variations in degree of absorption from the mucosa, was probable in man, and suggested that these findings had an important bearing on the passage of infective material from the nasal cavities to the brain.

It is also interesting to note that, during experimental work on poliomyelitis, Faber (1933) demonstrated that the hairs of the olfactory nerves lie free in the olfactory portion of the nasal mucosa and are covered only by mucus. In spite of this it is important to note that intranasal application of the poliomyelitis virus into the nares of monkeys does not invariably lead to the development of experimental infection even though the monkey is a relatively susceptible animal (Fairbrother and Hurst, 1930). The poliomyelitis virus passes to the central nervous sytem along the axons of the olfactory nerve. This is a different route from that described above, but the area of nasal mucosa at which the neural tract is entered is probably the same in both cases.

There is thus reliable evidence of definite but complex communication tract ween the nasal cavities and the central nervous system; these are situated in the olfactory portion of the nasal mucosa and occupy a relatively small area on the septal and lateral walls of the superior nasal meatus. Passage of infective material along the olfactory tract is not, however, readily accomplished.

Applying these observations to the pathogenesis of meningococcal meningitis, the following hypothesis is postulated.

The meningococcus is widely disseminated by droplet infection and gains entrance to the upper respiratory tract by the nostrils and mouth. The subsequent sequence of events is dependent on many factors, particularly important being the virulence and the number of the organisms, their implantation on the olfactory portion of the nasal mucosa, and the subsequent overpowering of the local defence mechanism.

In the majority of individuals these requirements are not fulfilled; the meningococcus becomes a transient member of the pharyngeal flora, probably as a commensal, and the individual becomes a temporary carrier. Under circumstances particularly favourable to the meningococcus, the local defence barrier is overcome and the organisms may, by direct extension, reach either the theca or possibly the blood capillaries; in the latter event the septicaemic type of infection might result.

Many factors are concerned with the tendency for outbreaks to appear under war-time conditions. During the war 1939-45 opportunities for the dissemination of the meningococcus were greatly increased as a result of overcrowding and poor ventilation, which tended to be accentuated by black-out precautions, while in certain groups, such as new recruits, general resistance was probably lowered by fatigue and exposure, especially during the winter. It is also interesting to note that Group I strains were responsible for the main outbreak and there is much indirect evidence to indicate that these strains are more virulent than Group II.

A possible explanation for occurrence of sporadic Group II infections, found mainly in infants and children, during inter-epidemic periods is the close contact of infants with adults, in particular mothers and nurses, and the consequent greater risk of frequent and large dosage with these strains. Adults may possess some form of specific resistance to the meningococcus, but there is no convincing evidence that the development of specific immunity plays a major role in the defence against meningococcal infections. It is, however, possible that the local defence mechanism is less efficient in infants than in adults, and is more readily overpowered by the relatively avirulent Group II strains.

\section{Summary}

The results of this investigation do not support the theory that meningococcal infections occur in three successive stages-catarrhal, septicaemic, and meningitic. Support is given to the theory of direct spread of infection from the nasopharynx to the theca by the perineural sheaths of the olfactory nerves.

I am indebted to many colleagues, in particular Dr. D. C. Liddle, for assistance in the collection of material for this investigation, and wish to offer my sincere thanks for their help. 


\section{REFERENCES}

Andrewes, F. W., Bulloch, W., and Hewlett, R. T. (1916). Med. Res. Cncl. Sp. Rep. Ser., No. 2. P. 29.

Banks, H. S. (1939). Lancet, 921.

Banks, H. S. (1947). Bull. Min. Hlth. Publ. Hlth. Lab. Serv., 6, 138.

Banks, H. S. (1947). Bull. Min. Hlth. Publ. Hlth. Lab. So

Beeson, P. B., and Westerman, E. (1943). Brit. med. J., 1, 497

Branham, S. E. (1940). Bact. Rev., 4, 59.

Brinton, D. (1941). "Cerebrospinal Fever." Livingstone, Edin-

, W. Le Gros. (1929). Min. Health Rep. Publ. Hith. Med. Sub., No. 54

Cleminson, F. J. (1918). Brit. med. J., 2, 51 Dopter, C. (1921). "L'Infection Méningococcique." Ballière et Fils,

Dudley, S. F., and Brennan, J. R. (1934). J. Hyg., Camb., 34, 525.

Embleton, D., and Steven, G. H. (1919). Lancet, 2, 682.

Faber, H. K. (1933). Medicine, 12, 83.

Fairbrother, R. W. (1940). Brit. med. J., $2,859$.

Fairbrother, R. W., and Hurst, E. W. (1930). J. Path. Bact., 33, 17.

Fildes, P., and Baker, S. L. (1918). Med. Res. Cncl. Sp. Rep. Ser. No. 17.

Flack, M. (1917). Med. Res. Cncl. Sp. Rep. Ser., No. 3, p. 31.

Foster, M., and Gaskell, J. F. (1916). "Cerebrospinal Fever," University Press, Cambridge.
Glover, J. A. (1920). Med. Res. Cncl. Sp. Rep. Ser., No. 50, p. 133

Gordon, M. H., and Murray, E. G. (1915). J. R.A.M.C., $25,411$.

Griffith, F. (1918). J. Hyg., Camb., 17, 124

Harries, G. E. (1942). Brit. med. J., 2, 423.

Herrick, W. W. (1918). J. Amer. med. Ass., 71, 612

Jubb, A. A. (1943). Brit. med. J., 1, 501

Logan, W. R. (1946). Edinb. med. J., 53, 183, 235.

Lundie, A., Thomas, D. J., and Fleming, S. (1915). Brit. med. J., 1,

Ministry of Health Memorandum No. 234 (1940). “Cerebrospinal Fever."

Ministry of Health (1946). "Report of Chief Medical Officer, 19391945."

Murray, E. G. D. (1929). Med. Res. Cncl. Sp. Rep. Ser., No. 124.

Priest, R. (1941). J. R.A.M.C., 76, 249.

Rolleston, Sir H. (1919). Lancet, 1, 541, 593, 645.

Scott, W. M. (1918). J. Hyg., Camb., 17, 191.

Straker, E Hill, A. B., and Lovell, R. (1939). Rep. Pub. Hlth. Med. Sub. No. 90 .

Strong, P. S. (1943). Amer. J. med. Sci., 206, 561.

War Office Memorandum (1940 and 1942). "Cerebrospinal Fever among Troops."

Worster-Drought, C. C., and Kennedy, A. M. (1919). “Cerebrospinal Fever." Black, Ltd., London. 